\title{
Physical activity among Iranian physicians and faculty members: A cross sectional study
}

\section{Shahram Mohaghegh}

Assistant Professor of Sports and Exercise Medicine, Clinical Research Development Center of Loghman Hakim Hospital, Shahid Beheshti University of Medical Sciences, Tehran, Iran

\begin{abstract}
As many physicians and faculty members of educational hospitals need to do their tasks in out of hospitals, it seems to be vulnerable to sedentary life style. Sedentary lifestyle has been associated with many chronic conditions and recognized as a leading cause of total mortality. It can affect their educational tasks too. As there is little evidences about this topic, we decided to investigate the physical activity level of physicians and faculty members. Physical activity level of 80 physicians and faculty members of Loghman Hospital which is an educational hospital of Shahid Beheshti university of medical sciences was measured by self-completing of international physical activity questionnaire (IPAQ). Also they are asked about their sex, age, marriage status, degree and type of it, academic ranking, being a faculty member or not and part time or fulltime employment. Data was analysed with SPSS16 software with appropriate statistical tests. Physical activity level of physicians and faculty members of Loghman Hospital was in the range of low to moderate (less than $3000 \mathrm{MET} /$ minute per week). Except for type of educational degree, other variables were not associated significantly with their physical activity levels. Physical activity of emergency physicians, anaesthesiologist and surgeons was significantly higher than of internists. Promotion for increasing physical activity and exercise of physicians in the university are recommended.
\end{abstract}

KEY WORDS: PHYSICAL ACTIVITY; EXERCISE; FACULTY MEMBERS; PHYSICIAN; IRAN

ARTICLE INFORMATION:

*Corresponding Author: sh.mohaghegh@sbmu.ac.ir

Received $29^{\text {th }}$ April, 2017

Accepted after revision $30^{\text {th }}$ Sep, 2017

BBRC Print ISSN: 0974-6455

Online ISSN: 2321-4007 CODEN: USA BBRCBA

\% Thomson Reuters ISI ESC and Crossref Indexed Journal

NAAS Journal Score 2017: 4.31 Cosmos IF: 4.006

- A Society of Science and Nature Publication, 2017. All rights reserved.

Online Contents Available at: http//www.bbrc.in/

DOI: $10.21786 / \mathrm{bbrc} / 10.4 / 29$ 


\section{INTRODUCTION}

There is the strong evidence that physical activity (PA) is a crucial determinant of health. The definition of Physical activity is "any bodily movement produced by skeletal muscles that result in energy expenditure". Exercise is a subcategory of physical activity which is planned, structured, repetitive, and purposive for improvement or maintenance of one or more components of physical fitness, (Caspersen, Powell et al. 1985). Sedentary lifestyle has been associated with many chronic conditions and recognized as a leading cause of total mortality in the modern era, (Mohaghegh and Hajian 2015, Castro, Ng et al. 2017).

Having physical activity and exercise is considered as primary prevention against 35 chronic non communicable conditions [including accelerated biological aging/ premature death, low cardiorespiratory fitness ( $\left.\mathrm{VO}_{2} \max \right)$, sarcopenia, metabolic syndrome, obesity, insulin resistance, prediabetes, type 2 diabetes, non-alcoholic fatty liver disease, coronary heart disease, peripheral artery disease, hypertension, stroke, congestive heart failure, endothelial dysfunction, arterial dyslipidemia, hemostasis, deep vein thrombosis, cognitive dysfunction, depression and anxiety, osteoporosis, osteoarthritis, balance, bone fracture/falls, rheumatoid arthritis, colon cancer, breast cancer, endometrial cancer, gestational diabetes, preeclampsia, polycystic ovary syndrome, erectile dysfunction, pain, diverticulitis, constipation, and gallbladder diseases, (Booth, Roberts et al. 2012, Hajain Mohaghegh et al. 2017).

It is estimated that non communicable diseases (NCD) will account for 73\% of deaths and 60\% of the global disease burden by 2020. Cardiovascular disease, cancer, and diabetes are now among the most prevalent, costly, and preventable of all health problems (Rao, Darshan et al. 2012). It has been considered that (9\%) of premature deaths in 2008 worldwide were related with sedentary life style. Determinants of physical inactivity includes insufficient time, injuries, obesity, and lack of encouragement by family and friends and environmental factors such as air pollution and lack of facilities, (Shahram Mohaghegh, Ramin Kordi et al. 2012, Mandil, Alfurayh et al. 2016).

Available data in Iran shows that national and regional inactivity among Iranian people is a serious and prevalent issue. Reported inactivity ranged from approximately $30 \%$ to $70 \%$ with considerable variation between sexes and studied sub-groups, (Fakhrzadeh, Djalalinia et al. 2016). However, there is a lack of data from Iran, especially among some sub-population groups. Physicians and faculty members are one of such subgroups .They are highly educated and may be more engaged in physical activity than others. On the other hand as many physicians and faculty members of edu- cational hospitals need to do their tasks in out of hospitals, it seems to be vulnerable to sedentary life style. As there is little evidences about this topic, we decided to investigate the physical activity level of physicians and faculty members of Loghman hospital which is an educational hospital of Shahid Beheshti University of Medical Sciences in Tehran, Iran.

\section{MATERIAL AND METHODS}

In the current cross-sectional study, after proper instruction and obtaining written consent, the Persian-translated long form self-completed International Physical Activity Questionnaire (IPAQ) was used for assessing physical activity level of 80 physicians and faculty members of Loghman Hospital in Tehran, Iran in summer 2017. The validity and reliability of this version of IPAQ have already been proven in Iranian sample of individuals (Vasheghani-Farahani, Tahmasbi et al. 2011). The results were expressed as low (less than 600 metabolic equivalent (MET). minute per week), medium (between 600 to 3000 MET. minute per week), and high (more than 3000 MET. minute per week) level of physical activity. There was no limitation in data gathering and all physicians and faculty members of the hospital who participated in the research were questioned. Also they were asked about their sex, age, marriage status, degree and type of it, academic ranking, being a faculty member or not and part time or fulltime employment in University. Data was analysed with SPSS16 software with appropriate statistical tests.

\section{RESULTS AND DISCUSSION}

Totally, 80 doctors and faculty members were evaluated: 26 females (32.5\%) and 54 males (67.5\%) with the average age of 44.2, minimum 30 and maximum 72 years. Other epidemiologic characteristics of patients are provided in Table 1.

Mean of physical activity level of all participants were 429.98 MET. Minute/week. A total of 58 individuals (72.5\%) had low physical activity level, 15 individuals (18.8\%) medium, and no one (0\%) had high physical activity level. There was no data about physical activity level of 7 persons. There were not significant differences in age, sex, marriage status, duration of employments, being a faculty member or not, and academic ranking for faculty members between persons with low and medium physical activity levels ( $p$ value more than 0.05). There was significant difference in terms of different physical activity levels between the doctors and faculty members who were internist (not surgeon) and surgeons, anaesthesiologists and emergency medicine 


\begin{tabular}{|c|c|c|c|}
\hline Variable & Description & Number & Percent \\
\hline \multirow{2}{*}{ Marriage status } & Single & 9 & 11.5 \\
\hline & Married & 71 & 88.5 \\
\hline \multirow{4}{*}{ Educational Degree } & General Practitioners (GPs) & 2 & 2.5 \\
\hline & Internists (not surgeons) & 47 & 58.8 \\
\hline & Surgeons & 20 & 25 \\
\hline & $\begin{array}{l}\text { anaesthesiologists and } \\
\text { emergency medicine specialists }\end{array}$ & 10 & 13 \\
\hline \multirow{2}{*}{ Being a faculty member } & yes & 64 & 79.7 \\
\hline & No & 16 & 20.3 \\
\hline \multirow{3}{*}{$\begin{array}{l}\text { Academic Ranking of } \\
\text { faculty members }\end{array}$} & Assistant Professor & 44 & 54.4 \\
\hline & Associate Professor & 14 & 17.7 \\
\hline & Full Professor & 6 & 7.6 \\
\hline \multirow{4}{*}{ Duration of employment } & Less than or equal 10 years & 46 & 58.1 \\
\hline & $11-20$ years & 15 & 18.9 \\
\hline & 21-30 years & 14 & 17.6 \\
\hline & More than 30 years & 5 & 5.4 \\
\hline
\end{tabular}

specialists(p value less than 0.014), which means in low physical activity group internists had relatively higher percentage (70 percent) than their surgeon, anaesthesiologist and emergency medicine specialist counterparts (30 percent). Totally there was significant difference in physical activity level of internists(non surgeons) and surgeons, anaesthesiologists and emergency medicine specialists ( $\mathrm{P}$ value less than 0.027) (Table 2). The highest physical activity level was related to anaesthesiologists and emergency medicine specialists, surgeons, Internists and GPs respectively.

According to the findings of the present study, mean of physical activity level of physicians and faculty members were 429.98 MET. Minute/week which was considered in the range of low physical activity level. Most (72.5 percent) of the participants in our study had low physical activity level and only 18.8 percent had medium physical activity level. These findings are consistent with data of some national and province-based studies about sedentary life style in Iranian people,
(Janghorbani, Amini et al. 2008), (Alikhani, Delavari et al. 2009) and (Janghorbani, Amini et al. 2007). However, there are some studies which reported higher levels of physical activity in Iran (Esteghamati, Khalilzadeh et al. 2011). These differences may be due to using different questionnaire which makes understudy comparison difficult or different studied sub-populations.

One study with similar participants which was conducted in emergency medical personnel in Hamedan Province of Iran, reported the prevalence of low, medium and high physical activity levels to be $73.5 \%, 24 \%$ and $2 \%$ respectively which is very close to our prevalence rate, (Gharlipour Gharghani, Sayarpour et al. 2011).

In the present study, there was not any significant difference in gender ratio of persons with low and medium physical activity levels. This is in contrast of most of data about physical activity levels of women in Iran which indicates lower physical activity of Iranian women compared to men, (Hajain Mohaghegh et al. 2017). The possible explanation for this difference

Table 2. Mean of physical activity levels of doctors and faculty members according to type of speciality of them

\begin{tabular}{l|l|l|l|}
\hline Type of degree & Mean (MET minute/week) & Number & Std. Deviation \\
\hline General Practitioners (GP) & 190.0000 & 1 &. \\
Internists & 287.8444 & 45 & 307.35588 \\
Surgeons & 608.3333 & 18 & 657.19323 \\
Emergency medicine specialists & 632.2222 & 9 & 468.98235 \\
and anaesthesiologists & 429.9863 & 73 & 458.41770 \\
Total & & &
\end{tabular}


may be that female physicians and faculty members are highly educated and acknowledge the benefits of physical activity and exercise. Another important finding in our study was significant lower physical activity level of internists compared to anaesthesiologists and emergency medicine specialists. Maybe the working lifestyle of these physicians makes them more physically active.

There are some data about higher physical activity of physicians in some countries. A national study in Saudi Arabia 2015 showed that $65.2 \%$ of primary health care physicians were performing moderate to vigorous physical activities while $34.8 \%$ of them were physically inactive, (Lobelo and de Quevedo 2016).

Another study conducted in female family doctors in Estonia 2010 indicated that 92\% of doctors have moderate to high level of physical activity, (Suija, Pechter et al. 2010). It seems that for the physicians and faculty members, lack of time was the most important reason for not practicing in physical activities and exercise. This is believable and indicated in previous studies as the most important reason of sedentary life style among physicians (Bahram, Abbas et al. 2003). As physicians who are faculty members have more working hours and related responsibilities it is expected to have sedentary lifestyle. On the other hand, increasing the physical activity of physicians and faculty members can enhance their counselling role about physical activity and exercise, improve job satisfaction and reduce rates of depression and finally improve patient care (Williams, Williams et al. 2015). This study has some limitations. First, the study was done in just one educational hospital and so the results cannot be expanded. Second, physical activity level of participants was measured subjectively by self-completing of the questionnaire which can be different between persons. It is proposed for future studies to include more hospitals and educational centers and use objective tools for measuring physical activity (such as pedometer).

\section{CONCLUSION}

As sedentary lifestyle is common among physicians and faculty members, promotion for increasing physical activity and exercise in this group is recommended. This can enhance their health and increase their job satisfaction and productivity. Also increasing the number of physicians and faculty members in educational hospitals can provide enough time for them to practice more physical activity and exercise.

\section{ACKNOWLEDGMENTS}

The fund of this study was provided by research proposal number 4590 of Shahid Beheshti University of medical sciences, Clinical Research Development Center of Loghman Hakim Hospital. The author gratefully acknowledges Professor Latif Gachkar and Dr Maryam Hajian for their contribution and assistance of the statistical analysis and Mrs Effat Barari for her contribution in data collection.

\section{CONFLICT OF INTEREST}

There is no conflict of interest.

\section{REFERENCES}

Alikhani, S., Delavari, A., Alaedini, F., Kelishadi, R., Rohbani, S. and Safaei, A. (2009). A province-based surveillance system for the risk factors of non-communicable diseases: A prototype for integration of risk factor surveillance into primary healthcare systems of developing countries. Public Health 123(5): 358-364.

Bahram, S., Abbas, B., Kamal, J. and Fakhro, E. (2003). Leisuretime physical activity habits among physicians. Bahrain Med Bull 25(2): 80-82.

Booth, F. W., Roberts, C. K. and Laye, M. J. (2012). Lack of exercise is a major cause of chronic diseases.Comprehensive Physiology 2(2): 1143-1211.

Caspersen, C. J., Powell, K. E. and Christenson, G. M. (1985). Physical activity, exercise, and physical fitness: definitions and distinctions for health-related research.Public Health Reports 100(2): 126-131.

Castro, O., Ng, K., Novoradovskaya, E., Bosselut, G. and Hassandra, M. (2017). A scoping review on interventions to promote physical activity among adults with disabilities." Disability and health journal.

Esteghamati, A., Khalilzadeh, 0., Rashidi, A., Kamgar, M., Meysamie, A. and Abbasi, M. (2011). Physical activity in Iran: results of the third national surveillance of risk factors of noncommunicable diseases (SuRFNCD-2007). J Phys Act Health 8(1): 27-35.

Fakhrzadeh, H., Djalalinia, S., Mirarefin, M., Arefirad, T., Asayesh, H., Safiri, S., Samami, E., Mansourian, M., Shamsizadeh, M. and Qorbani, M. (2016). "Prevalence of physical inactivity in Iran: a systematic review." Journal of Cardiovascular and Thoracic Research 8(3): 92-97.

Gharlipour Gharghani, Z., Sayarpour, S. and Moeini, B. (2011). "Associated factors with regular physical activity among emergency medical personnel in Hamadan: Applying Health Belief Model." J Health Syst Res 7(6): 710-717.

Hajain M, Mohaghegh S, Ghachkar L and E, B. (2017). Investigations on the physical activity level of some Iranian drug suicidal patients.Biosci. Biotech. Res. Comm 10(3): 419423.

Janghorbani, M., Amini, M., Rezvanian, H., Gouya, M. M., Delavari, A., Alikhani, S. and Mahdavi, A. (2008). Association of body mass index and abdominal obesity with marital status in adults. Arch Iran Med 11(3): 274-281. 
Janghorbani, M., Amini, M., Willett, W. C., Mehdi Gouya, M., Delavari, A., Alikhani, S. and Mahdavi, A. (2007). First nationwide survey of prevalence of overweight, underweight, and abdominal obesity in Iranian adults. Obesity (Silver Spring) 15(11): 2797-2808

Lobelo, F. and de Quevedo, I. G. (2016). The evidence in support of physicians and health care providers as physical activity role models.American journal of lifestyle medicine 10(1): 36-52.

Mandil, A. M., Alfurayh, N. A., Aljebreen, M. A. and Aldukhi, S. A. (2016). Physical activity and major non-communicable diseases among physicians in Central Saudi Arabia.Saudi Med J 37(11): 1243-1250.

Mohaghegh, S. and Hajian, M. (2015). Stroke in a Young Swimmer. Asian Journal of Sports Medicine 6(2): e23812.

Rao, C. R., Darshan, B., Das, N., Rajan, V., Bhogun, M. and Gupta, A. (2012). Practice of Physical Activity among Future Doctors: A Cross Sectional Analysis. Int J Prev Med 3(5): 365-369.
Mohaghegh S, Kordi R, Younesian M, Hajian M and Mohaghegh B (2012). Investigating the trend of hourly changes of air pollutants in Tehran during 2004 to 2008 and exercise recommendations for athletes and patients. Pajoohande 17(5): 234-240.

Suija, K., Pechter, Ü., Maaroos, J., Kalda, R., Rätsep, A., Oona, M. and Maaroos, H.-I. (2010). Physical activity of Estonian family doctors and their counselling for a healthy lifestyle: a cross-sectional study. BMC family practice 11(1): 48.

Vasheghani-Farahani, A., Tahmasbi, M., Asheri, H., Ashraf, H., Nedjat, S. and Kordi, R. (2011). The Persian, Last 7-day, Long form of the International Physical Activity Questionnaire: Translation and Validation Study.Asian Journal of Sports Medicine 2(2): 106-116.

Williams, A. S., Williams, C. D., Cronk, N. J., Kruse, R. L., Ringdahl, E. N. and Koopman, R. J. (2015). Understanding the exercise habits of residents and attending physicians: a mixed methodology study. Family medicine 47(2): 118-123. 